\title{
Caracterización de la fisiología restrictiva del ventrículo derecho mediante flujo 4D
}

\footnotetext{
Julián Vega ${ }^{1}$, Daniel Lorenzatti ${ }^{2}$, Silvia Montserrat ${ }^{2}$, José Tomas Ortiz-Pérez ${ }^{2}$, Adelina Doltra ${ }^{2}$, Rosario J Perea ${ }^{3}$, Teresa M de Caralt ${ }^{3}$, Santiago Sotes ${ }^{4}$, Susana Prat-González $z^{2}$.

1. División de Enfermedades Cardiovasculares. Escuela de Medicina. Pontificia Universidad Católica de Chile. Santiago, Chile 2. Institut Clínic Cardiovascular, Hospital Clínic; Institut d'Investigacions Biomèdiques August Pi i Sunyer (IDIBAPS), Barcelona, España 3. Institut Clínic de Diagnòstic per la Imatge, Hospital Clinic de Barcelona; Institut d'Investigacions Biomèdiques August Pi i Sunyer (IDIBAPS), Barcelona, España.

4. Institut Clínic de Diagnòstic per la Imatge, Hospital Clinic de Barcelona, España.
}

\section{4-D flow characterization of right ventricular restrictive physiology}

Right ventricular restrictive physiology (RVRP) occurs in diverse clinical scenarios, most frequently after repair of Tetralogy of Fallot (TOF). Cardiac magnetic resonance (CMR) can comprehensively evaluate RVRP using 4D flow along with anatomical and fibrosis characterization. Also, RVRP is associated with less pulmonary regurgitation and fewer right ventricle enlargement; its long term protective role is debated. RVRP is a challenging and relevant diagnosis, which hallmark is the presence of antegrade pulmonary arterial flow in late diastole throughout the respiratory cycle. Also, other hemodynamic findings could aid such us flow in; caval veins, suprahepatic, coronary sinus and tricuspid valve. Obtaining all these flow curves is virtually impossible by echocardiography. CMR with $4 \mathrm{DF}$ is a unique and powerful technique enabling this comprehensive hemodynamic evaluation as depicted in this case. Keywords: Right Ventricular Dysfunction, Pulmonary artery, Regional Blood Flow, Tetralogy of Fallot 
La fisiología restrictiva del ventrículo derecho (FRVD) se presenta en diversos escenarios clínicos, siendo frecuente encontrarla en el seguimiento de las cardiopatías congénitas, específicamente en la Tetralogía de Fallot (TdF) reparada, donde se presenta en más del $50 \%$ de los pacientes ${ }^{1}$ y se ha asociado a fibrosis extensa del tracto de salida ${ }^{2}$. La Resonancia Magnética Cardíaca (RMC), ofrece la posibilidad de combinar una evaluación completa y dirigida de los flujos y gradientes de presión mediante técnicas de flujo en 4 dimensiones (flujo-4D) junto con la caracterización de la fibrosis miocárdica es una técnica con gran potencial.

Clásicamente, la FRVD ha sido relacionada con menor regurgitación pulmonar y dilatación del ventrículo derecho (VD) y mejor capacidad funcional ${ }^{3}$. Sin embargo, su papel protector a largo plazo es actualmente debatido ${ }^{1}$. En cuanto a su diagnóstico, los parámetros utilizados difieren de la clásica evaluación diastólica del ventrículo izquierdo, debido a que el flujo tricúspideo es muy variable y sensible a condiciones de carga, siendo menos fiable cuando el llenado ventricular proviene simultáneamente desde la aurícula derecha (AD) y la arteria pulmonar (AP), como ocurre en presencia de insuficiencia pulmonar significativa. Por lo tanto, para su diagnóstico se utiliza un hallazgo particular que consiste en la presencia de flujo diastólico anterógrado (FDA) en la AP que, para ser considerado patológico, debe estar presente durante todo el ciclo respiratorio. Este fenómeno refleja la baja distensibilidad del VD, que limita su adaptación al volumen diastólico, lo que se traduce en un aumento considerable de la presión, superando la presión diastólica de la AP causando apertura prematura de la válvula con la consecuente eyección anterógrada tele diastólica, también llamado FDA 4 .

Si bien en la FRVD la presencia de FDA es el hallazgo cardinal, existen otras alteraciones hemodinámicas en la circulación derecha que es posible evaluar de forma exhaustiva mediante RMC utilizando flujo-4D. En la Figura 1 se muestran mediciones de flujo del corazón derecho a diferentes niveles mediante una única adquisición con flujo-4D en un paciente de 40 años con una TdF reparada que presenta FRVD.

\section{- Flujo en la Vena Cava Superior}

A medida que aumenta la disfunción diastólica, el 1lenado sistólico disminuye causando que la onda $S$ (onda "x" del pulso venoso) sea inferior a la onda D (onda "y" del pulso venoso). Además, después de la contracción auricular derecha se observa una reversión de flu- jo mayor a lo normal.

\section{- Flujo Tricuspídeo}

A diferencia del corazón izquierdo, en la FRVD es menos frecuente tener un patrón restrictivo con $\mathrm{E} / \mathrm{A}>2.5$ y tiempo de desaceleración corto. Sin embargo, en este caso se observa una onda E prominente con una onda A muy pequeña y además una onda L' que refleja marcado aumento de la presión de la AD con flujo en la diástasis.

\section{- Flujo en la vena suprahepática}

En la FRVD el flujo de las venas suprahepáticas se caracteriza por la reversión de flujo diastólico (RF) ya descrita, por una onda sistólica (S) menor a la diastólica (D) y también puede registrarse una onda $\mathrm{V}$ profunda, como se ve también en la insuficiencia tricúspidea severa.

\section{- Flujo en el seno coronario}

El flujo en el seno coronario es similar al flujo venoso central. Se pone de manifiesto una onda "v mayor a la onda "a" (a diferencia de la fisiología constrictiva en la cual se igualan), una onda "a" prominente y una onda " $y$ " con un descenso marcado (DIP).

\section{- Flujo en la arteria pulmonar}

Al final de la diástole tras la contracción de la AD la presión del VD supera la presión de la AP generando apertura prematura de la válvula y FDA (Figura 1 panel superior derecho, flecha blanca). Es importante resaltar que la contracción auricular no contribuye al llenado del VD, ya que éste se comporta como un tubo rígido que transmite el volumen entre la AD y la AP. Conviene recordar que, si bien en condiciones normales puede observarse un breve FDA durante la inspiración, la adquisición del flujo-4D se realiza en respiración libre, y la curva obtenida es un promedio de distintos ciclos cardíacos y respiratorios. Por lo tanto, se considera que un FDA evaluado mediante flujo-4D es patológico cuando supera el $2.5 \%$ del volumen anterógrado ${ }^{2}$.

\section{Conclusión}

Si bien la presencia de FDA en la AP se considera un equivalente de FRVD, este hallazgo requiere una evaluación e interpretación cuidadosa. En este sentido, la RMC, en particular las técnicas de evaluación hemodinámica como el flujo-4D, tienen un gran potencial para caracterizar de forma no invasiva la fisiología cardíaca en situaciones complejas, como es la FRVD en el seguimiento de las cardiopatías congénitas. 


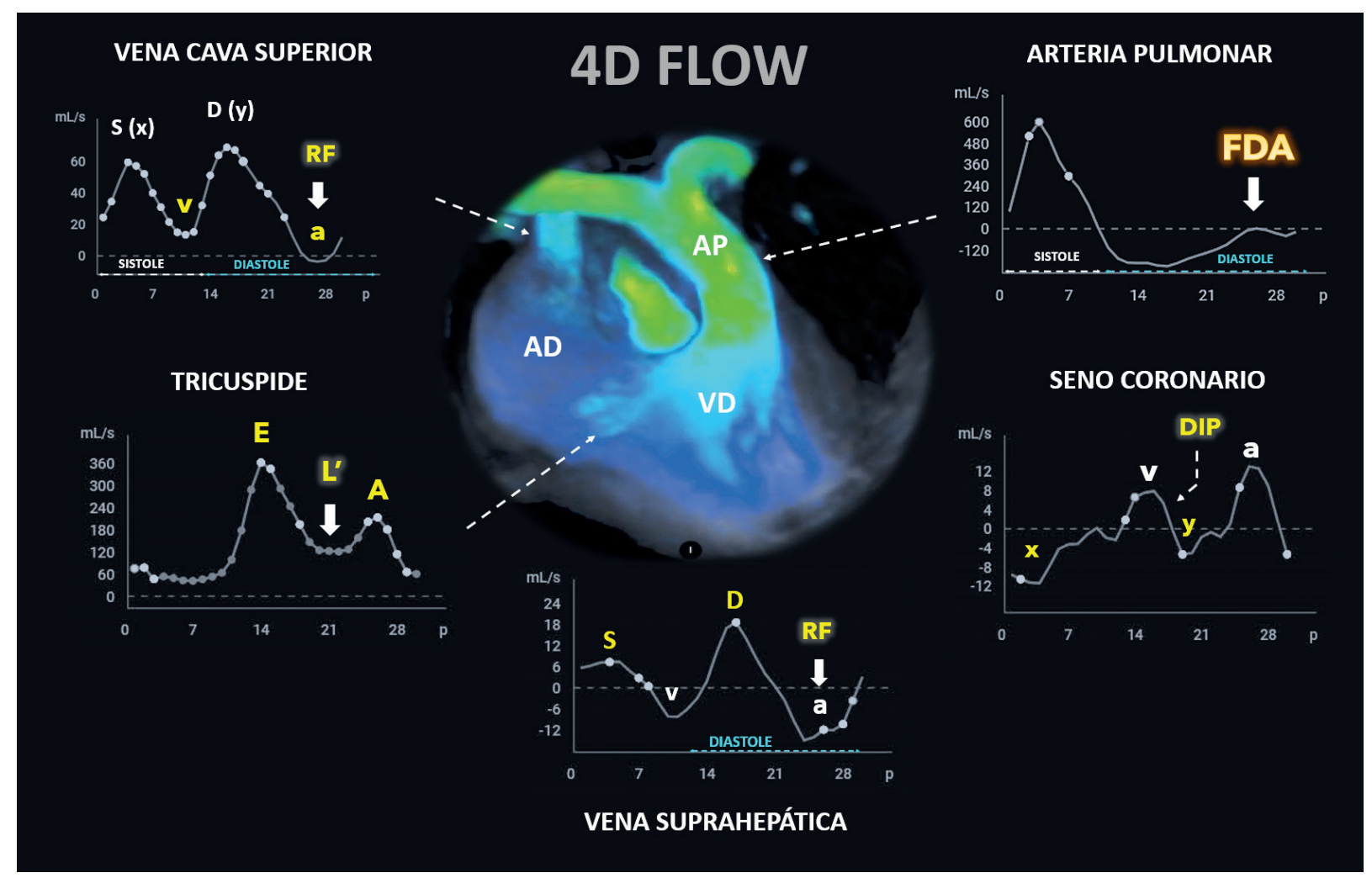

AD: Aurícula derecha, VD: Ventrículo derecho, AP: Arteria pulmonar, DIP: Descenso marcado de la onda " $y$ ". FDA: Flujo diastólico anterógrado, RF: Reversión de flujo, Sístole frames 29-11, Diástole frames 12-28.

\section{Referencias}

1. MERCER-ROSA L, FOGEL MA, PARIDON SM, RYCHIK J, YANG W, GOLDMUNTZ E. Revisiting the End-Diastolic Forward Flow (Restrictive Physiology) in Tetralogy of Fallot. JACC Cardiovasc Imaging. 2018;11(10):1547-1548.

2. Munkhammar P, Carlsson M, Arheden H, Pesonen E. Restrictive right ventricular physiology after Tetralogy of Fallot repair is associated with fibrosis of the right ventricular outflow tract visualized on cardiac magnetic resonance imaging. Eur Hear J - Cardiovasc Imaging. 2013;14(10):978-985.
3. GATZOULIS MA, CLARK AL, CULLEN S, NEWMAN CGH, REDINGTON AN. Right Ventricular Diastolic Function 15 to 35 Years After Repair of Tetralogy of Fallot. Circulation. 1995;91(6):1775-1781.

4. KUTTY S, VALENTE AM, WHITE MT, et al. Usefulness of Pulmonary Arterial End-Diastolic Forward Flow Late After Tetralogy of Fallot Repair to Predict a "Restrictive" Right Ventricle. Am J Cardiol. 2018;121(11):1380-1386. 